\title{
PERIODIC TRAVELLING WAVES
}

\author{
V. HUTSON \\ Department of Applied Mathematics \\ The University of Sheffield \\ Sheffield S3 7RH, United Kingdom \\ K. MISCHAIKOW \\ Center for Dynamical Systems and Nonlinear Studies \\ School of Mathematics, Georgia Institute of Technology \\ Atlanta, Georgia 30332, U.S.A. \\ E-mail: mischaik@math.gatech.edu
}

\begin{abstract}
Some new techniques for finding periodic travelling waves are discussed.
1. Introduction. It is our belief that periodic travelling waves are a common phenomena for a wide class of ecological models and situations with bistablility are of particular interest as the direction of the wave gives information as to the 'winning' species. To be more precise, recall that for the classical models for pairs of interacting species, either mutualist, competitive, or predator-prey, there are variety of proofs demonstrating the existence of monotone bistable travelling waves $[1,2,3,5,7,8]$. Suppose now that another species, a competitor, predator or prey, is introduced and this new species can as its numbers change reverse the 'dominance' of the existing species. Then, if the reaction time of the new species is slow we expect a periodic travelling wave to exist. This paper is concerned with the special case of mutualist species under the inclusion of a predator which does not diffuse. The paper will be used to demonstrate the potential of a singular perturbation theory based on the Conley index.
\end{abstract}

A general model for all these problems is

$$
\frac{\partial u_{1}}{\partial t}=\mu_{1} \frac{\partial^{2} u_{1}}{\partial x^{2}}+u_{1} f_{1}\left(u_{1}, u_{2}, w\right)
$$

1991 Mathematics Subject Classification: Primary 35K57; Secondary 92D25.

This work was supported in part by NATO grant 930149. The second author was also partially supported by NSF grants DMS-9505116 and DMS-9805584.

The paper is in final form and no version of it will be published elsewhere. 


$$
\begin{aligned}
\frac{\partial u_{2}}{\partial t} & =\mu_{2} \frac{\partial^{2} u_{2}}{\partial x^{2}}+u_{2} f_{2}\left(u_{1}, u_{2}, w\right) \\
\frac{\partial w}{\partial t} & =\delta \frac{\partial^{2} u_{2}}{\partial x^{2}}+\epsilon w g\left(u_{1}, u_{2}, w\right)
\end{aligned}
$$

where it is assumed that $0<\delta<<\epsilon<<1$ and $f_{i}, g \in C^{2}\left(\mathbf{R}^{3}, \mathbf{R}\right)$. In this system $u_{1}$ and $u_{2}$ represent the mutualist, competitive, or predator-prey species. $w$ is the third species which interacts slowly with respect to $u_{1}$ and $u_{2}$. Since $\delta, \epsilon \approx 0, \partial w / \partial t \approx 0$, and hence on short time scales the behavior of the ecological system will be governed by

$$
\begin{aligned}
& \frac{\partial u_{1}}{\partial t}=\mu_{1} \frac{\partial^{2} u_{1}}{\partial x^{2}}+u_{1} f_{1}\left(u_{1}, u_{2}, w\right) \\
& \frac{\partial u_{2}}{\partial t}=\mu_{2} \frac{\partial^{2} u_{2}}{\partial x^{2}}+u_{2} f_{2}\left(u_{1}, u_{2}, w\right)
\end{aligned}
$$

where $w$ is constant. The question that will be addressed here is the long term effects of the additional species $w$ under the assumption that (2) only admits monotone bistable waves.

Travelling wave solutions to (1) take the form

$$
\left(u_{1}(x, t), u_{2}(x, t), w(x, t)\right)=\left(u_{1}(x+\theta t), u_{2}(x+\theta t), w(x+\theta t)\right) .
$$

This gives rise to the following system of ordinary differential equations:

$$
\begin{aligned}
u_{1}^{\prime} & =p_{1} \\
\mu_{1} p_{1}^{\prime} & =\theta p_{1}-u_{1} f_{1}\left(u_{1}, u_{2}, w\right) \\
u_{2}^{\prime} & =p_{2} \\
\mu_{2} p_{2}^{\prime} & =\theta p_{2}-u_{2} f_{2}\left(u_{1}, u_{2}, w\right) \\
w^{\prime} & =q \\
\delta q^{\prime} & =\theta q-\epsilon g\left(u_{1}, u_{2}, w\right) .
\end{aligned}
$$

The assumption that $0<\delta<<\epsilon<<1$ suggests considering the following formal series of reductions. Setting $\delta=0$ and solving for $q$ transforms (3) to

$$
\begin{aligned}
u_{1}^{\prime} & =p_{1} \\
\mu_{1} p_{1}^{\prime} & =\theta p_{1}-u_{1} f_{1}\left(u_{1}, u_{2}, w\right) \\
u_{2}^{\prime} & =p_{2} \\
\mu_{2} p_{2}^{\prime} & =\theta p_{2}-u_{2} f_{2}\left(u_{1}, u_{2}, w\right) \\
w^{\prime} & =\frac{\epsilon}{\theta} g\left(u_{1}, u_{2}, w\right) .
\end{aligned}
$$

Now setting $\epsilon=0$ leads to the further reduction that $w^{\prime}=0$ and hence $w$ can be treated as a parameter:

$$
\begin{aligned}
u_{1}^{\prime} & =p_{1} \\
\mu_{1} p_{1}^{\prime} & =\theta p_{1}-u_{1} f_{1}\left(u_{1}, u_{2}, w\right) \\
u_{2}^{\prime} & =p_{2} \\
\mu_{2} p_{2}^{\prime} & =\theta p_{2}-u_{2} f_{2}\left(u_{1}, u_{2}, w\right) .
\end{aligned}
$$


Observe that (5) is the travelling wave system for (2). The assumptions concerning the existence of the monotone bistable travelling waves can be presented in terms of the reaction system

$$
\begin{aligned}
& u_{1}^{\prime}=u_{1} f_{1}\left(u_{1}, u_{2}, w\right) \\
& u_{2}^{\prime}=u_{2} f_{2}\left(u_{1}, u_{2}, w\right),
\end{aligned}
$$

and for this paper are as follows:

M1 The species $u_{i}$ are mutualistic, e.g. $\partial f_{i} / \partial u_{j}>0(i \neq j)$. Furthermore, intraspecific competition holds, e.g. $\partial f_{i} / \partial u_{i}<0$.

M2 There exists $w_{c}>0$ such that for all $w \in\left[0, w_{c}\right]$ the system (6) has exactly three equilibria $A=(0,0), B(w)=\left(b_{1}(w), b_{2}(w)\right)$ and $C(w)=\left(c_{1}(w), c_{2}(w)\right)$ with $0<$ $c_{i}(w)<b_{i}(w) \leq 1$. All three equilibria are hyperbolic; $A$ and $B(w)$ are stable and $C(w)$ has a one-dimensional unstable manifold.

M3 For all nonnegative values of $w, A$ is a global attractor for the flow on the axis, $u_{1}=0$ or $u_{2}=0$, that is, the mutualism is obligate.

Under these assumptions the existence of monotone travelling waves independent of the diffusion coefficients has been established [7]. More precisely, for any $w$ there exists a wave speed $\theta$ for which there is a solution $\left(u_{w}(t), p_{w}(t)\right)$ of $(5)$ with the property that

$$
\lim _{t \rightarrow-\infty}\left(u_{w}(t), p_{w}(t)\right)=(A, 0) \text { and } \lim _{t \rightarrow \infty}\left(u_{w}(t), p_{w}(t)\right)=(B(w), 0)
$$

where $0=(0,0)$ in the $p_{1}-p_{2}$ plane. Furthermore, it is known that these waves are unique [5]. Therefore, it makes sense to let $\theta(w)$ denote the wave speed at which there exists a bistable travelling wave solution to (5). Since the nonlinearities are smooth and since the waves are unique, $\theta$ is smooth.

We shall now make the following assumption:

$\Theta \quad \theta(0)$ and $\theta\left(w_{c}\right)$ are of opposite sign.

We shall define

$$
K:=\min \left\{|\theta(0)|,\left|\theta\left(w_{c}\right)\right|\right\} .
$$

It should be pointed out that there are few techniques for determining precisely the wave speed for bistable waves in systems. Therefore, one of the contributions of this paper is to provide coarse estimates for the values of the wave speed. This will be described in Section 4.

Before stating the general results we need to provide hypotheses on the reaction terms of the third species $w$. The simplest model for $w$ predating on the $u_{i}$ is

W1 $u_{i} f_{i}\left(u_{1}, u_{2}, w\right)=u_{i}\left(F_{i}\left(u_{1}, u_{2}\right)-w\right), \quad i=1,2$.

Under this assumption it will be shown in Section 3 that $\theta$ is a monotone decreasing function of $w$.

The final assumption is that the predator population decreases or increases in the absence or presence of sufficient prey.

W2 For all $w \geq 0, g(A, w)<0$ and for $w \in\left[0, w_{c}\right], g(B(w), w)>0$.

We are finally in a position to state one of the results of this paper. 
Theorem 1. Given M1 - M3, $\Theta$, W1 - W2, and $\theta \in(0, K)$, for $0 \leq \epsilon<<1$, sufficiently small, there exists a periodic travelling wave.

As will become clear in the next section, this theorem follows directly from a much more general result presented in [4]. In Section 4 we will discuss the more difficult question of how to verify the hypothesis $\Theta$. It should also be remarked that $\mathbf{W} \mathbf{1}$ is not essential given $\Theta$ and some regularity on the function $\theta(w)$. However, we leave this argument to another paper.

2. Existence of periodic orbits. In this section we shall present an extremely simple version of [4, Corollary 1.8] which will be used to establish Theorem 1. Since we believe it will be of use in other applications we present it in an abstract form.

Consider the system

$$
\begin{array}{ll}
\dot{\xi}=f(\xi, \eta), & \xi \in \mathbf{R}^{n} \\
\dot{\eta}=\epsilon g(\xi, \eta) & \eta \in \mathbf{R}
\end{array}
$$

Since $0<\epsilon<<1$ it is natural to consider the one-parameter family of equations

$$
\dot{\xi}=f(\xi, \eta), \quad \eta \in[0, \bar{a}] .
$$

For fixed $\eta$, let $\varphi_{\eta}: \mathbf{R} \times \mathbf{R}^{n} \rightarrow \mathbf{R}^{n}$ denote the flow generated by (8).

We shall make the following assumptions.

B1 There are continuous functions $\alpha, \beta:[0, \bar{a}] \rightarrow \mathbf{R}^{n}$ such that $\alpha(\eta)$ and $\beta(\eta)$ are hyperbolic equilibria under $\varphi_{\eta}$, i.e. $D f(\alpha(\eta), \eta)$ and $D f(\beta(\eta), \eta)$ have no purely imaginary eigenvalues. Furthermore, the number of eigenvalues with positive real part are the same for $D f(\alpha(\eta), \eta)$ and $D f(\beta(\eta), \eta)$.

B2 There are constants $0<a_{0}<a_{1}<\bar{a}$ and compact sets $N_{0}, N_{1} \subset \mathbf{R}^{n}$ with the following properties.

1. For $\eta \in\left[0, a_{0}\right]$ :

(a) $N_{0}$ is an isolating neighborhood under $\varphi_{\eta}$.

(b) $\alpha(\eta), \beta(\eta) \in N_{0}$.

(c) If $\xi \in \operatorname{Inv}\left(N_{0}, \varphi_{\eta}\right)$ and $\xi \neq \alpha(\eta) \cup \beta(\eta)$, then the omega limit set of $\xi$ under $\varphi_{\eta}$ is $\beta(\eta)$ and the alpha limit set of $\xi$ under $\varphi_{\eta}$ is $\alpha(\eta)$.

2. For $\eta \in\left[a_{1}, \bar{a}\right]$ :

(a) $N_{1}$ is an isolating neighborhood under $\varphi_{\eta}$.

(b) $\alpha(\eta), \beta(\eta) \in N_{1}$.

(c) If $\xi \in \operatorname{Inv}\left(N_{1}, \varphi_{\eta}\right)$ and $\xi \neq \alpha(\eta) \cup \beta(\eta)$, then the omega limit set of $\xi$ under $\varphi_{\eta}$ is $\alpha(\eta)$ and the alpha limit set of $\xi$ under $\varphi_{\eta}$ is $\beta(\eta)$.

3. For $\eta \in\left\{0, a_{0}\right\}$,

$$
\operatorname{Inv}\left(N_{0}, \varphi_{\eta}\right)=\{\alpha(\eta), \beta(\eta)\}
$$

4. For $\eta \in\left\{a_{1}, \bar{a}\right\}$,

$$
\operatorname{Inv}\left(N_{1}, \varphi_{\eta}\right)=\{\alpha(\eta), \beta(\eta)\}
$$

5. The topological transition matrices $T_{0, a_{0}}$ and $T_{a_{1}, \bar{a}}$ do not equal the identity.

B3 For $\eta \in[0, \bar{a}], g(\alpha(\eta), \eta)<0$ and $g(\beta(\eta), \eta)>0$. 
TheOREM 2 (4, [Corollary 1.8]). Under assumptions B1-B3, for $\epsilon>0$ but sufficiently small there exists a periodic solution to (7).

Proof of Theorem 1. The assumption B1 is satisfied by choosing $\alpha(w)=(A, 0)$ and $\beta(w)=(B(w), 0)$.

B2.1 and B2.2 follow from the construction of an isolating neighborhood in [7] and $\Theta$. B2.3 and B2.4 follow from the monotonicity of $\theta(w)$ (this will be proven in the next section). Finally, the proof of the existence of the monotone bistable wave for fixed values of $w$ given in [7] involved verifying B2.5.

3. Monotonicity of the wave speed. As was indicated above W1 implies the monotonicity of the wave speed as a function of predator population. In biological terms this is a rather natural observation: the larger the predation, the greater the tendency for the mutualists to die out, and so the smaller $\theta$ is.

In what follows superscripts 1,2 denote quantities corresponding to given constant levels $w^{(1)}$ and $w^{(2)}$ of the predator.

LEMma 3. $w^{(2)}>w^{(1)}$ implies that $\theta^{(2)}<\theta^{(1)}$.

Proof. This is based on a standard comparison theorem for the quasi-monotone reaction system (see [6] for example). Let $\left(u_{1}^{(i)}\left(x+\theta^{(i)} t\right),\left(u_{2}^{(i)}\left(x+\theta^{(i)} t\right)\right)\right.$ be traveling wave solutions. For the equilibrium $B(w)$ it is clear that $b_{j}\left(w^{(2)}\right)<b_{j}\left(w^{(1)}\right)$ for $j=1,2$. Note next that for $j=1,2$,

$$
\begin{aligned}
u_{j t}^{(1)}-u_{j}^{(1)}\left(F\left(u_{1}^{(1)}, u_{2}^{(1)}\right)-w^{(2)}\right)-\mu_{j} u_{j x x}^{(1)} & \geq u_{j t}^{(1)}-u_{j}^{(1)}\left(F\left(u_{1}^{(1)}, u_{2}^{(1)}\right)-w^{(1)}\right)-\mu_{j} u_{j x x}^{(1)} \\
& =0 .
\end{aligned}
$$

This shows that $\left(u_{1}^{(1)}, u_{2}^{(1)}\right)$ is a supersolution for the $w^{(2)}$ equation. A linearization argument, for which the details are omitted, shows that as $z \rightarrow-\infty$, for $j=1,2$

$$
u_{j}^{(2)}(z)<u_{j}^{(1)}(z) .
$$

We can thus arrange (by translating time if necessary) that

$$
u_{j}^{(2)}(x)<u_{j}^{(1)}(x) .
$$

It follows from the comparison theorem that for $t>0$

$$
u_{j}^{(2)}\left(x+\theta^{(2)} t\right)<u_{j}^{(1)}\left(x+\theta^{(2)} t\right)
$$

Therefore, the $w^{(1)}$ wave cannot overtake the $w^{(2)}$ wave and the result follows.

4. Estimating wave speeds. Of all the hypotheses required for Theorem 1, the most difficult to verify is $\Theta$ since this requires obtaining bounds on $\theta(w)$. Here we outline a simple method applicable to a limited class of equations, but we intend to consider more general methods in a more complete account to be published elsewhere.

Suppose $h \in C^{1}\left(\left[0, b_{1}(w)\right] \times\left[0, b_{2}(w)\right], \mathbf{R}\right)$. Then, differentiation and use of the governing ordinary differential equations yields the relation 


$$
\begin{aligned}
h(B)-h(0)=\theta & \left(\int_{0}^{b_{1}(w)} p_{1}(s) d s+\int_{0}^{b_{2}(w)} p_{2}(s) d s\right) \\
& +\int_{0}^{b_{1}(w)}\left(\frac{\partial h}{\partial u_{1}}-f_{1}\right) d u_{1}+\int_{0}^{b_{2}(w)}\left(\frac{\partial h}{\partial u_{2}}-f_{2}\right) d u_{2} .
\end{aligned}
$$

Since the bistable wave is monotone increasing, $p_{i} \geq 0$. There are, also, upper bounds on these quantities given in terms of the $\mu_{i}$ and $f_{i}$ (see [7]). It follows that if

$$
\frac{\partial h}{\partial u_{i}} \leq f_{i}
$$

and $h(B)-h(0)>0$, then $\theta>0$. Furthermore, $\theta<0$, if the appropriate inequalities are reversed. The question is, of course, whether we can find a suitable function $h$.

This method works easily for the following particular example. Suppose that

$$
f_{1}\left(u_{1}, u_{2}, w\right)=f_{2}\left(u_{2}, u_{1}, w\right)
$$

with

$$
f\left(u_{1}, u_{2}, w\right)=u_{1}\left(-1-10 u_{1}^{3}+10.5 u_{2}^{2}+0.5 v-k w\right) .
$$

Take

$$
h\left(u_{1}, u_{2}\right):=-\frac{\gamma}{2}\left(u_{1}^{2}+u_{2}^{2}\right)-2\left(u_{1}^{5}+u_{2}^{5}\right)+5.25 u_{1}^{2} u_{2}^{2}
$$

with $\gamma=1$ if $k=0$ and $\gamma=1$ if $k=0.9$. A simple check shows that this gives negative and positive $\theta$ respectively.

Acknowledgements. We would like to thank Glen Vickers for discussions concerning Section 4

\section{References}

[1] C. Conley and R. Gardner, An application of the generalized Morse index to travelling waves solutions of a competative reaction diffusion model, Ind. Univ. J. 33 (1979), 319-343.

[2] R. GARDNER, Existence and stability of travelling wave solutions of competition models: a degree theoretic approach, JDE 44 (1982).

[3] R. Gardner, Existence of travelling wave solution of predator prey systems via the Conley index, SIAM J. Appl. Math. 44 (1984), 56-76.

[4] T. Gedeon, H. Kokubu, K. Mischaikow, H. Oka, and J. Reineck, The Conley index for fast-slow systems I: One-dimensional slow variables, J. Diff. Eq. Dyn., to appear.

[5] W. Huang, Uniqueness of bistable traveling wave for mutualist species, preprint 1998.

[6] A. W. Leung, Systems of Nonlinear Partial Differential Equations, Kluwer, Dordrecht, 1989.

[7] K. Mischaikow and V. Hutson, Travelling waves for mutualist species, SIAM J. Math. Anal. 24 (1993), 987-1008.

[8] K. Mischaikow and J. Reineck, Travelling waves in predator prey systems, SIAM J. Math. Anal. 24 (1993). 